\title{
Gradual Damping of Solitary Waves
}

\author{
By Garbis H. Keulegan
}

\begin{abstract}
This paper treats the problem of the damping by viscous action of translation waves. A shcrt exposition is given of Boussinesq's boundary layer theory for wave motion, and expressions for the damping of rectangular and solitary waves are derived. Scott Russell's experimental results for solitary waves are compared with the theory, and satisfactory agreement is found to exist. This fact makes it legitimate to apply the formulas developed to correct in model tests on harbors or in other tests of a like nature for the dissipative effects that occur in shallow-water waves.
\end{abstract}

\section{List of Symbols}

$A=\omega h_{1} / H$.

$B=$ breadth of channel.

$e=$ base of natural logarithms.

$d E_{1} / d t=$ rate of dissipation of energy of the wave.

$d E_{2} / d t=$ rate of decrease of energy of the wave.

$\delta E_{1}=$ energy in column of unit length and of width $d x$.

$F(\quad)=$ function of.

$g=$ acceleration of gravity.

$h$ or $h_{1}=$ height of wave above undisturbed surface.

$h_{01}=$ initial height of wave above undisturbed surface.

$H=$ depth of undisturbed water.

$k=$ constant defined by eq 35 .

$K=$ constant defined by eq 44 .

- $L=$ length of wave.

$n=k x$.

$N=$ an integral defined by eq 41 .

$p=$ the pressure in the liquid.

$q=$ a variable.

$s=$ a variable, also the distance travelled by the wave.

$t=$ time.

$x=$ coordinate parallel to solid surface in direction of motion.

$y=$ coordinate parallel to solid surface at right angles to direction of motion.

$z=$ coordinate perpendicular to solid surface.

$u, v, w=$ velocity components of particles in the boundary layer parallel to $x, y, z$, respectively.

$u_{0}, v_{0}, w_{0}=$ velocity components of particles in the potential region parallel to $x, y, z$, respectively.

$V=$ particle velocity in the potential region.

$X=x$-component of body force per unit mass due to gravity.

$Y=y$-component of body force per unit mass due to gravity.

$Z=z$-component of body force per unit mass due to gravity.

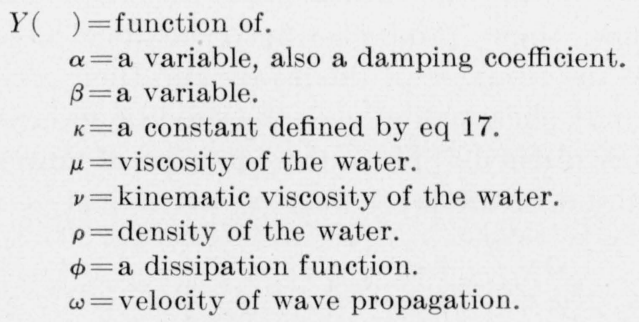

\section{Introduction}

In the propagation of translation waves in still water, the main source of dissipation of the energy is the thin layer of liquid next to the solid boundaries of the channel; that is, in the boundary layer. The motion in the layer is laminar, and the determination of the velocities in it can be made by resorting to the ordinary equations of viscous motion. The motion of the liquid outside of the layer is potential, and there the values of the velocities are exactly those that would be obtained from the ordinary theory, assuming that the laminar layer is absent. The errors involved in the use of this method can be ignored, since the variation of pressure in a cross section of the laminar layer is negligible. In fact, the gradual decrease of height of a solitary wave, the only wave of translation, which under ideal conditions, travels without change of form, can be explained on the basis of this dissipation, at least, when the wave is of moderate proportions. The purpose of this paper is to develop a method of computing this energy dissipation and to compare the results with ex- 
perimental data as a means of verifying the accuracy of the theory.

\section{Dynamical Equation of the Laminar Layer}

The theory of the laminar layers with reference to wave motion was initiated by Boussinesq, and the essential elements of his method will now be given [1]. ${ }^{1}$

Consider the unsteady motion of a liquid near a flat solid boundary. For the sake of generality it will be supposed that the motion is two-dimensional. We select the rectangular system of axes $x, y, z$, where $z$ is normal to the solid surface. The velocity components of particles along these axes are $u, v$, and 0 , respectively. At the solid boundary, that is, at $z=0, u$ and $v$ vanish. In the motion of waves $u, \partial u / \partial x$, and similar quantities are small, and hence their products may be neglected. Owing to the thinness of the layers and their great lengths, only shears parallel to the solid boundaries are of consequence. Thus, the equation of motion for the liquid in the layer may be written as

and

$$
\left.\begin{array}{c}
\frac{\partial u}{\partial t}=-\frac{1}{\rho} \frac{\partial p}{\partial x}+X+\nu \frac{\partial^{2} u}{\partial z^{2}}, \\
\frac{\partial v}{\partial t}=-\frac{1}{\rho} \frac{\partial p}{\partial y}+Y+\nu \frac{\partial^{2} v}{\partial z^{2}}, \\
0=-\frac{1}{\rho} \frac{\partial p}{\partial z}+Z,
\end{array}\right\}
$$

where $\nu$ is the kinematic viscosity, $\rho$ is the density of the liquid, $p$ is the pressure, and $X, Y, Z$ are the components of the body forces per unit mass due to gravity. The third equation indicates that in the boundary layer the pressure $p$, the part due to disturbances, does not vary along the normals to the boundary. The pressure, $p$, in the viscous layer is the same function of $x$ and $y$ as in the potential region next to the viscous layer.

Denoting the limiting values of $u$ and $v$ at the boundary of the layer by $u_{0}$ and $v_{0}$, these last being the velocities in the potential region, we have for this region

$$
\left.\begin{array}{l}
\frac{\partial u_{0}}{\partial t}=-\frac{1}{\rho} \frac{\partial p}{\partial x}+X \\
\frac{\partial v_{0}}{\partial t}=-\frac{1}{\rho} \frac{\partial p}{\partial y}+Y .
\end{array}\right\}
$$

\footnotetext{
${ }_{1}^{1}$ Figures in brackets indicate the literature references at the end of this paper.
}

Subtracting these equations from the corresponding ones in eq1, we obtain

$$
\frac{\partial\left(u-u_{0}\right)}{\partial t}=\nu \frac{\partial^{2}\left(u-u_{0}\right)}{\partial z^{2}}
$$

and

$$
\frac{\partial\left(v-v_{0}\right)}{\partial t}=\nu \frac{\partial^{2}\left(v-v_{0}\right)}{\partial z^{2}}
$$

with the boundary conditions

$$
\left.\begin{array}{ll}
u-u_{0}=-u_{0} & z=0 \\
u-u_{0}=0 & z=\infty
\end{array}\right\}
$$

and

$$
\left.\begin{array}{ll}
v-v_{0}=-v_{0} & z=0 \\
v-v_{0}=0 & z=\infty
\end{array}\right\} .
$$

These are the basic boundary equations as derived by Boussinesq. In the form given above they are applicable to a progressive wave of oscillation in a channel or to a standing wave in a rectangular basin.

The limiting value, $z=\infty$, appearing in the boundary conditions is introduced for convenience in the mathematical analysis. Physically the boundary layer is very thin, and the velocities $u$ and $v$ attain the values $u_{0}$ and $v_{0}$, respectively, at small finite distances away from the solid boundaries. Moreover, if the waves examined are progressive oscillatory waves moving in a rectangular channel and the layer next to the bottom is considered, the axis of $z$ is taken vertical and the $x$ axis is in the same direction as the channel axis. For this case $v=0$, and $u_{0}$ is a function of $x, z$, and $t$. When the boundary layer of one of the vertical walls is considered, the axis of $z$ is taken normal to the wall, the axis of $y$ is vertical, and, as before, the axis of $x$ is parallel to the channel axis. Now $u_{0}$ and $v_{0}$ are the velocities in the potential region outside of the layer and are functions of $x, y$, and $t$ only. The case of the standing waves in a rectangular channel or in a rectangular basin is interpreted in the same manner.

As we shall be interested primarily in translation waves, we need consider only

$$
\frac{\partial\left(u-u_{0}\right)}{\partial t}=\nu \frac{\partial^{2}\left(u-u_{0}\right)}{\partial z^{2}}
$$


with the boundary conditions

$$
\left.\begin{array}{ll}
u-u_{0}=-u_{0} & z=0 \\
u-u_{0}=0 & z=\infty
\end{array}\right\} .
$$

Now $u_{0}$ is the uniform velocity in the cross section and is a function of $x$ and $t$, assuming that the wave travels in the direction of $x$ positive or $x$ negative.

In the approximate theory of waves, if $h$ is the superelevation of the wave surface, measured from the undisturbed water surface, and $H$ is the depth of the water in the channel, for a wave traveling in the direction of $x$ positive:

$$
u_{0}=\omega \frac{h}{H},
$$

where $\omega$ is the velocity of wave propagation given by

$$
\omega=\sqrt{g H} .
$$

For a wave of translation having any arbitrary form but a small wave height, and for a solitary wave, independently of height, for waves traveling in the direction of $x$ positive, we have

$$
h=h(x-\omega t),
$$

and

$$
u=u_{0}(x-\omega t) .
$$

The meaning of these expressions is that the shape of waves does not change with translation. Obviously there are two methods available for the description of the waves. We may observe the variation of the wave height with time at a fixed point $x(x=0)$, or we may observe the variation of the wave height with $x$ at a fixed time $t(t=0)$.

We select the second method of observation, and then the main problem resolves itself into determining the velocities in the boundary layer in the entire length of the wave at a given time, $t$. From this point of view, the basic equation now is, replacing $\partial / \partial t$ in eq 7 by $-\omega(\partial / \partial x)$

$$
\frac{\partial\left(u-u_{0}\right.}{\partial x}=-\frac{\nu}{\omega} \frac{\partial^{2}\left(u-u_{0}\right)}{\partial z^{2}},
$$

with the boundary conditions

$$
\left.\begin{array}{ll}
u-u_{0}=-u_{0} & z=0 \\
u-u_{0}=0 & z=\infty
\end{array}\right\}
$$

In general, for $t$ constant, it will be assumed that

$$
u_{0}=F(x) .
$$

\section{Distribution of the Velocities in the Vis-} cous Layer.

The expression

$$
u-u_{0}=-e^{-z} \sqrt{\frac{\kappa \omega}{2 \nu}} \cos \left[\kappa(\alpha-x)-z \sqrt{\frac{\kappa \omega}{2 \nu}}\right],
$$

where $\kappa$ and $\alpha$ are independent of $z$ and $x$, as is well known, is a solution of eq 13 and satisfies the second equation of the boundary conditions, eq 14 . Putting $z=0$, and remembering that $u=0$ at $z=0$, we obtain

$$
u_{0}=\cos [\kappa(\alpha-x)] .
$$

Thus we conclude that when the velocity of $u_{0}$ in the potential region of the water is periodic and is given by eq 17 , velocity $u$ in the laminar layer is given by eq 16 .

To obtain the velocities in the viscous layer when the wave consists of a long intumescence, we require a general solution with the value of $u_{0}$ given by eq 15. The general solution may be constructed from the elementary solution, eq 16. Multiplying its right-hand member by $F(\alpha) d \alpha$ and integrating between the limits, $+\infty$ and $-\infty$, we obtain

$u-u_{0}=-\int_{-\infty}^{+\infty} F(\alpha) e^{-z \sqrt{\frac{\kappa \omega}{2 \nu}}} \cos \left[\kappa(\alpha-x)-z \sqrt{\frac{\kappa \omega}{2 \nu}}\right] d \alpha$,

which still is a solution of eq 13 and satisfies the second condition of eq 14. Since the permissible values of $\kappa$ are positive numbers, we finally take

$$
\begin{aligned}
& u-u_{0}=-\frac{1}{\pi} \int_{0}^{\infty} d \kappa \int_{-\infty}^{+\infty} F(\alpha) e^{-z} \sqrt{\frac{\kappa \omega}{2 \nu}} \\
& \cos \left[\kappa(\alpha-x)-z \sqrt{\frac{\kappa \omega}{2 \nu}}\right] d \alpha,
\end{aligned}
$$

and this is the desired solution. For, when we make $z=0, u=0$, we have

$$
-u_{0}=-\frac{1}{\pi} \int_{0}^{\infty} d \kappa \int_{-\infty}^{+\infty} F(\alpha) \cos [\kappa(\alpha-x)] d \alpha,
$$

and, therefore, according to the rule of the Fourier integral, it gives $u_{0}=F(x)$, indicating that the first condition in eq 14 is also satisfied. 


\section{FIRATA}

"Gradual Damping of Solitary Waves" by Carbis it. Keuiegan

$$
\text { RPI }=5
$$

\section{Page}

489 Equation 13. Parenthesis mitted after $u_{n}$. 
Although eq 18 gives the distribution of velocities in the boundary layer for the most general case, it is desirable to transform the solution into a form more directly manageable for calculation. That is, it is better to change the double integral into a single integral by effecting an integration with respect to $\kappa$. With a view to making use of the two well-known definite integrals,

$$
\begin{aligned}
u-u_{0}= & -\frac{1}{\pi} \int_{-\infty}^{x} F(\alpha) d \alpha \int_{0}^{\infty} e^{-z \sqrt{\frac{\kappa \omega}{2 \nu}}} \cos \left[\kappa(\alpha-x)-z \sqrt{\frac{\kappa \omega}{2 \nu}}\right] d \kappa . \\
& -\frac{1}{\pi} \int_{x}^{+\infty} F(\alpha) d \alpha \int_{0}^{\infty} e^{-z \sqrt{\frac{\kappa \omega}{2 \nu}}} \cos \left[\kappa(\alpha-x)-z \sqrt{\frac{\kappa \omega}{2 \nu}}\right] d \kappa .
\end{aligned}
$$

In the first double integral in eq $21 \alpha \leqslant x$, and this suggests the transformations

$$
\begin{gathered}
\kappa(\alpha-x)=-2 s^{2} \\
z \sqrt{\frac{\kappa \omega}{2 \nu}}=q s,
\end{gathered}
$$

and

$$
d \kappa=\frac{4 s}{x-\alpha} d s .
$$

Introducing these transformations in the first double integral of eq 21 , the integral changes to

$$
-\frac{4}{\pi} \int_{-\infty}^{x} \frac{F(\alpha) d \alpha}{x-\alpha} \int_{0}^{\infty} e^{-q s} \cos \left(-2 s^{2}-q s\right) s d s,
$$

and therefore vanishes by virtue of eq 19. In the second double integral of eq $21, \alpha \leqslant x$, and this suggests the transformation

$$
\begin{gathered}
\kappa(\alpha-x)=2 s^{2}, \\
z \sqrt{\frac{\kappa \omega}{2 \nu}}=q s, \\
d \kappa=\frac{4 s}{\alpha-x} d s, \\
\alpha-x=\frac{\omega}{\nu} \frac{z^{2}}{q^{2}}
\end{gathered}
$$

and

$$
d \alpha=-\frac{2 \omega z^{2}}{\nu q^{3}} d q
$$

Introducing these transformations in the second double integral of eq 21 and making use of eq 20 , the final result is

$$
u-u_{0}=-\frac{1}{\pi} \int_{0}^{\infty} F\left(x+\frac{\omega z^{2}}{\nu q^{2}}\right) e \frac{-q^{2}}{4} d q,
$$

or putting $q=2 \beta$,

$$
u-u_{0}=-\frac{2}{\pi} \int_{0}^{\infty} F\left(x+\frac{\omega z^{2}}{4 \nu \beta^{2}}\right) e^{-\beta^{2}} d \beta,
$$

which is the desired solution and may be compared with the Duhamel solution of the heat-conduction equation [2].

\section{Loss of Energy in the Laminar Layer}

As it is assumed that the laminar layer in the vicinity of the solid boundaries is thin and of considerable length, it suffices to express the dissipation function in the form

$$
\phi=\mu\left(\frac{\partial u}{\partial z}\right)^{2}
$$

where $\mu$ is the viscosity of the liquid. Consider a rectangular column of indefinite height, the cross section being of unit length and of width $d x$. Denoting the rate of dissipation of energy in the column by $d / d t \delta E_{1}$,

$$
\frac{d}{d t}\left(\delta E_{1}\right)=\mu d x \int_{0}^{\infty}\left(\frac{\partial u}{\partial z}\right)^{2} d z .
$$

Now, since $u_{0}$ is independent of $z$,

$$
\int_{0}^{\infty}\left(\frac{\partial u}{\partial z}\right)^{2} d z=\int_{0}^{\infty}\left[\frac{\partial\left(u-u_{0}\right)}{\partial z}\right]^{2} d z .
$$

Integrating the right-hand member by parts, we have, since $u-u_{0}=-u_{0}$ when $z=0$, and $u-u_{0}=0$ when $z=\infty$,

$$
\int_{0}^{\infty}\left(\frac{\partial u}{\partial z}\right)^{2} d z=u_{0}\left(\frac{\partial u}{\partial z}\right)_{0}-\int_{0}^{\infty}\left(u-u_{0}\right) \frac{\partial^{2}\left(u-u_{0}\right)}{\partial z^{2}} d z
$$


where $(\partial u / \partial z)_{0}$ is the gradient of velocity at the wall. In view of eq 13 ,

$$
\int_{0}^{\infty}\left(\frac{\partial u}{\partial z}\right)^{2} d z=u_{0}\left(\frac{\partial u}{\partial z}\right)_{0}+\frac{\omega}{\nu} \frac{\partial}{\partial x} \int_{0}^{\infty} \frac{\left(u-u_{0}\right)^{2}}{2} d z .
$$

Substituting in eq 23 ,

$$
\begin{aligned}
\frac{d}{d t}\left(\delta E_{1}\right)= & \mu d y d x u_{0}\left(\frac{\partial u}{\partial z}\right)_{0}+ \\
& \rho \omega d y d x \frac{\partial}{\partial x} \int_{0}^{\infty} \frac{\left(u-u_{0}\right)^{2}}{2} d z .
\end{aligned}
$$

When this expression is integrated between the limits $x=+\infty$ and $x=-\infty$, the result gives the rate of energy dissipation in the entire length of the laminar boundary layer. Denoting this loss by $d E_{1} / d t$, the integration gives

$$
\frac{d E_{1}}{d t}=+\mu d y \int_{-\infty}^{+\infty} u_{0}\left(\frac{\partial u}{\partial z}\right)_{0} d x
$$

since at the infinite distances, $x=\infty$ and $x=-\infty$, the velocities $u$ and $u_{0}$ both vanish. This formula is from Boussinesq [1]. The interpretation of the formula is that for the determination of the rate of dissipation of energy in the entire wave it is sufficient to consider the gradient of the laminar velocities at the solid boundary and the velocities in the region outside of the boundary layers.

This result can be extended to limited waves with a new interpretation. Let the posterior and anterior feet of the wave be at the points $x_{1}$ and $x_{2}$, respectively. Since the thickness of the viscous layer at the front of the wave; that is, at point $x_{2}$, is negligibly small, the expression for the rate of dissipation of energy for the limited wave per unit width of channel may now be written, since $u_{0}$ is independent of $z$, as

$\mu \int_{x_{1}}^{x_{2}} u_{0}\left(\frac{\partial u}{\partial z}\right)_{0} d x=\mu \int_{x_{1}}^{x_{2}} d x \int_{0}^{\infty}\left(\frac{\partial u}{\partial z}\right)^{2} d z+\rho \frac{\omega}{2} \int_{0}^{\infty} u_{1}^{2} d z$

where $u_{1}$ is the laminar velocity at the point $x_{1}$. The interpretation of the two integrals on the right-hand side of the equation is as follows. The first integral (double) represents the rate of energy dissipation in the laminar boundary layer under the wave. The second integral represents the kinetic energy left behind in the channel as the wave travels onward. As far as the wave is concerned, the energy left behind is lost, so that also in the case of a limited wave the integral on the left-hand side represents the rate of loss of energy of the wave per unit width of channel.

\section{Gradual Extinction of Rectangular Waves}

The rectangular wave constitutes the simplest example of a translation wave, and the application of the above theories to obtain the expression for the gradual damping of the wave with distance involves no difficulty. In a rectangular wave the superelevation is constant, and the wave length is finite.

Denote the constant superelevation of the wave by $h_{1}$, the length by $L$, and the particle velocities in the potential region by $V$. Assuming that the

wave is traveling in the direction of positive $x$, we have

$$
u_{0}=V=\omega \frac{h_{1}}{H} ; \omega=\sqrt{g H} .
$$

Suppose that at time $t=0$, the front of the wave is at the station $x=L$ (see fig. 1). Then for the

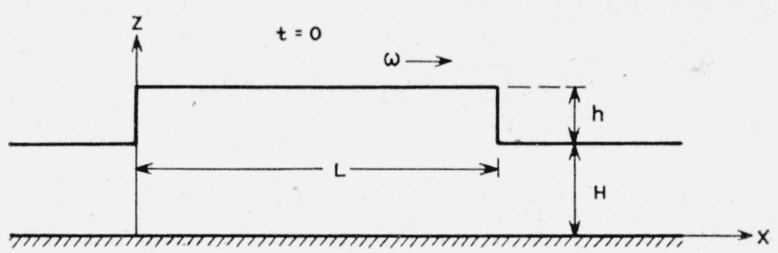

Figure 1. Surface profile of rectangular wave

description of the function $F$ appearing in eq 15 , we have

$$
\begin{array}{ll}
u_{0}=F(x)=0, & x \geqq L, \\
u_{0}=F(x)=V, & 0 \leqq x \leqq L, \\
u_{0}=F(x)=0, & x \leqq 0 .
\end{array}
$$

Accordingly, using eq 22, we find the boundary layer velocities to be

$$
u-V=-\frac{2 V}{\sqrt{\pi}} \int_{\frac{z}{2} \sqrt{\frac{\omega}{\nu(L-x)}}}^{\infty} e^{-\beta^{2}} d \beta,
$$

when $x$ lies in the range 0 to $L$, and

$u=-\frac{2 V}{\sqrt{\pi}} \int_{\frac{2}{2} \sqrt{\frac{\omega}{\nu(L-x)}}}^{\infty} e^{-\beta^{2}} d \beta+\frac{2 V}{\sqrt{\pi}} \int_{\frac{2}{2} \sqrt{\frac{\omega}{-\nu x}}}^{\infty} e^{-\beta^{2}} d \beta$,

when $x<0$. Since, in the present example, the 
rate of dissipation of the energy of the wave per unit width of channel reduces to

$$
\frac{d E_{1}}{d t}=\mu \int_{0}^{L} u_{0}\left(\frac{\partial u}{\partial z}\right)_{0} d x
$$

by the use of eq 23 , it is sufficient to consider only eq 27. Differentiating the latter equation with respect to $z$ and remembering that $V$ is independent of $z$,

$$
\frac{\partial u}{\partial z}=\frac{V}{\sqrt{\pi \nu(L-x) / \omega}}^{\frac{-z^{2} \omega}{4 \nu(L-x)}} .
$$

Putting $z=0$, the velocity gradient at the solid boundary is

$$
\left(\frac{\partial u}{\partial z}\right)_{0}=\frac{V}{\sqrt{\pi \nu(L-x) / \omega}} .
$$

Substituting in eq 24 and remembering that $u_{0}=+V$,

$$
\frac{d E_{1}}{d t}=\mu \int_{0}^{L} \frac{V^{2} d x}{\sqrt{\pi \nu(L-x) / \omega}} .
$$

Integrating and afterwards substituting from eq 25, we have finally

$$
\frac{d E_{1}}{d t}=\frac{2}{\sqrt{\pi}} \rho g^{5 / 4} H^{-3 / 4} h_{1}^{2} L^{1 / 2} \nu^{1 / 2},
$$

which is the value of the energy lost per unit time and per unit width of the channel. As was explained previously, the loss is made up of two parts. One part represents the dissipation of energy in the boundary layer under the wave; the other part represents the kinetic energy left in the liquid and in the vicinity of the solid boundary as the wave leaves a given station.

The energy of the wave, half potential and half kinetic, is given by

$$
E_{2}=g \rho h_{1}^{2} L,
$$

when computed for unit width of channel. The variation of the energy with the time is

$$
\frac{d E_{2}}{d t}=2 g_{\rho} h_{1} L \frac{d h_{1}}{a^{\prime} t} .
$$

Denoting the distance traveled by the wave by $s$,

$$
\frac{d h_{1}}{d t}=\frac{d h_{1}}{d s} \frac{d s}{d t}
$$

and as $d s / d t$ represents the velocity of the wave

$$
\frac{d h_{1}}{d t}=\sqrt{g H} \frac{d h_{1}}{d s} .
$$

Substituting this in eq 31 ,

$$
\frac{d E_{2}}{d t}=2 \rho g^{3 / 2} H^{1 / 2} L h_{1} \frac{d h_{1}}{d s} .
$$

By the principle of the conservation of energy, the decrease of the wave energy per unit time must equal the dissipation of energy per unit time. Accordingly the right-hand members of eq 30 and 32 are equal to each other in absolute value, but they are of opposite sign. With the grouping of the terms after the comparison is made, we have

$$
\left(\frac{h_{1}}{H}\right)^{-1} d\left(\frac{h_{1}}{H}\right)=-\frac{1}{\sqrt{\pi}}\left(\frac{L}{H}\right)^{-1 / 2} \sqrt{\frac{\mu}{g^{1 / 2} H^{3 / 2}}} d\left(\frac{s}{H}\right),
$$

which is the differential equation giving the rate of decrease of the wave height with the distance of travel. The equation was derived by assuming that the channel is very wide and the effect of the side walls could be neglected. If the channel is narrow, however, the second term in the righthand member must be multiplied by $(1+2 H / B)$, where $B$ denotes the width of the channel. That is, in a channel of finite width,

$$
\begin{aligned}
& \left(\frac{h_{1}}{H}\right)^{-1} d\left(\frac{h_{1}}{H}\right)=-\frac{1}{\sqrt{\pi}}(1+ \\
& \left.\frac{2 H}{B}\right)\left(\frac{L}{H}\right)^{-1 / 2}\left(\frac{\nu}{g^{1 / 2} H^{3 / 2}}\right)^{1 / 2} d\left(\frac{s}{H}\right) .
\end{aligned}
$$

The solution of the equation is, subject to the assumption, $h=h_{01}$ when $s=0$,

$$
\frac{h_{1}}{H}=\frac{h_{01}}{H} e^{-\alpha \frac{s}{H}},
$$

where

$$
\alpha=\frac{1}{\sqrt{\pi}}\left(1+\frac{2 H}{B}\right)\left(\frac{L}{H}\right)^{-1 / 2}\left(\frac{\nu}{g^{1 / 2} H^{3 / 2}}\right)^{1 / 2} .
$$

Accordingly, the decrease of wave height with distance is given by an exponential, and a logarithmic decrement that is independent of the height of the wave exists. For waves traveling in water of constant depth, the damping decreases with increase of wave length, with the increase of channel width, and with the decrease of kinematic viscosity. 
492 equation following eq 29, e omitted before exponent. Should read $e \frac{-z^{2} \omega}{4 \nu(I-x)}$

493 Second column. Equation for $\frac{\dot{y} u}{c^{t} \mathrm{z}}$.

$-\frac{k \omega}{4} \cdot \frac{z^{2}}{a^{2}}$ should read $\quad-\frac{k\left(a^{\prime}\right.}{4} \cdot \frac{z^{2}}{a^{2}}$. 


\section{Gradual Extinction of Solitary Waves}

Denoting the superelevation of the crest by $h_{1}$, the superelevation of the wave profile for $t$ constant $(t=0)$ is given approximately [3] by

$$
h=h_{1} \operatorname{sech}^{2} k x,
$$

where

$$
k=\frac{1}{H} \sqrt{\frac{3 h_{1}}{4 H}}
$$

The dimensionless plot of the wave profile, when the depth of water is twice the crest elevation, is shown in figure 2. Using eq 9 and 12, we may

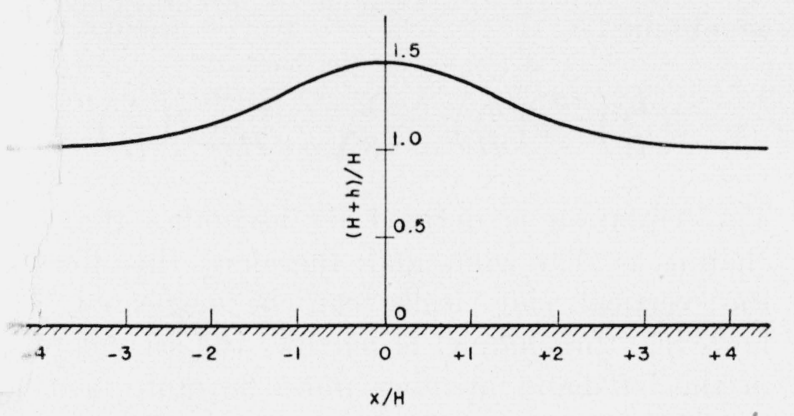

FIGURE 2. Surface profile of solitary wave, $\left(h_{1}=H / 2\right)$.

write for the particle velocities in the potential region the expressions, assuming that the wave is traveling in the direction of $x$ negative,

$$
u_{0}=A \operatorname{sech}^{2} k x ; A=\omega \frac{h_{1}}{H}
$$

In the region of the viscous boundary layers, using eq 22 ,

$$
u-u_{0}=-\frac{2 A}{\sqrt{\pi}} \int_{0}^{\infty} \operatorname{sech}^{2}\left(k x+\frac{k \omega z^{2}}{4 \nu \beta^{2}}\right) e^{-\beta^{2}} d \beta
$$

Differentiating with respect to $z$ and remembering that $u_{0}$ is independent of $z$,

$$
\begin{aligned}
\frac{\partial u}{\partial z}= & +\frac{4 A}{\sqrt{\pi}} \int_{0}^{\infty} \operatorname{sech}^{2}\left(k x+\frac{k \omega z^{2}}{4 \nu \beta^{2}}\right) \tanh (k x+ \\
& \left.\frac{k \omega z^{2}}{4 \nu \beta^{2}}\right) \frac{k \omega z}{2 \nu \beta^{2}} e^{-\beta^{2}} d \beta .
\end{aligned}
$$

The limiting value of the integral when $z$ approaches zero cannot be obtained directly, and a transformation of variables becomes necessary. Putting:

$$
k x=n,
$$

and

so that

$$
\sqrt{\frac{k}{4 \nu}} \frac{z}{\beta}=\alpha
$$

we have

$$
-\sqrt{\frac{k \omega}{4 \nu}} \frac{z}{\beta^{2}} d \beta=d \alpha,
$$

$$
\begin{aligned}
& \frac{\partial u}{\partial z}=+\frac{4 A}{\sqrt{\pi}} \sqrt{\frac{k \omega}{\nu}} \int_{0}^{\infty} \operatorname{sech}^{2}\left(n+\alpha^{2}\right) \tanh (n+ \\
&\left.\alpha^{2}\right) e^{-\frac{k \omega}{4} \frac{z^{2}}{\alpha^{2}}} d \alpha .
\end{aligned}
$$

This gives for $z=0$

$$
\left(\frac{\partial u}{\partial z}\right)_{0}=\frac{4 A}{\sqrt{\pi}} \sqrt{\frac{k \omega}{\nu}} \int_{0}^{\infty} \operatorname{sech}^{2}\left(n+\alpha^{2}\right) \tanh \left(n+\alpha^{2}\right) d \alpha .
$$

The rigorous determination of the definite integral appearing in the right-hand side is difficult, and sufficiently accurate results can be obtained if the indicated integration is replaced by the process of summation. Writing

$$
Y(n)=\int_{0}^{\infty} \operatorname{sech}^{2}\left(n+\alpha^{2}\right) \tanh \left(n+\alpha^{2}\right) d \alpha,
$$

\begin{tabular}{|c|c|c|c|}
\hline$n$ & $Y(n)$ & $n$ & $Y(n)$ \\
\hline 4.0 & 0.0000 & -0.2 & 0.1858 \\
\hline 3.0 & .0066 & -.4 & .0812 \\
\hline 2.5 & .0167 & -.6 & -.0187 \\
\hline 2. 0 & .0440 & -.8 & -.1017 \\
\hline 1. 6 & .0916 & -1.2 & -.1495 \\
\hline 1. 2 & .1725 & -1.6 & -.1408 \\
\hline 0.8 & .2988 & -2.0 & -.1132 \\
\hline .6 & .3526 & -2.5 & -.0820 \\
\hline .4 & .3798 & -3.0 & -.0598 \\
\hline .2 & .3639 & -4.0 & -.0355 \\
\hline .0 & .2975 & -5.0 & -.0243 \\
\hline
\end{tabular}

the desired summation for a specific $Y(n)$ was carried out by assigning to the interval $d \alpha$ the value 0.1 . The results of such determinations are given in table 1. The graph of $Y(n)$ is shown in figure 3 . Since $Y(n)$ is proportional to the velocity gradient at the channel bed, the curve may be interpreted as giving the variation of this velocity gradient along the wave. The velocity gradient at the wall reverses its sign at $n=-0.55$. The maximum value of the velocity gradient at $n=0.35$.

$$
\text { TABLE 1.-Numeri:al values of the function } Y(n)
$$




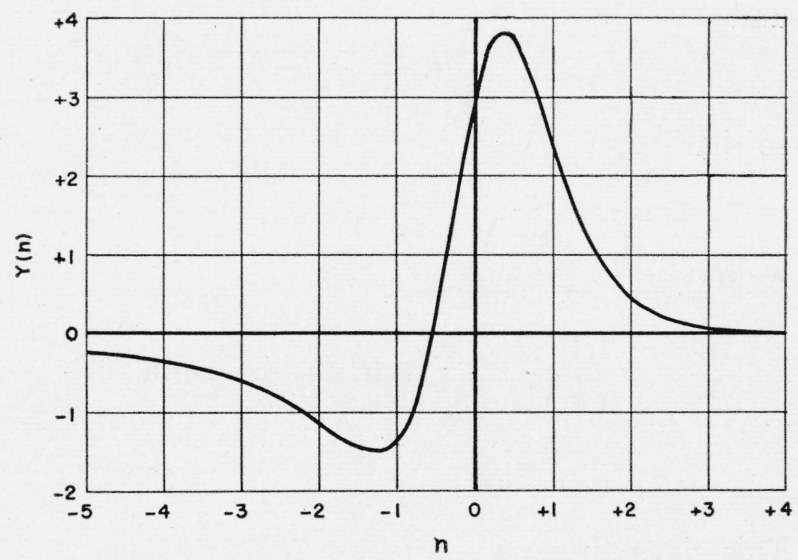

Figure 3. Variation of velocity gradient at wall with distance from center of wave.

For the case when the wave height is half of the undisturbed water depth, the reversal occurs at the point, $x=-0.9 H$. The maximum occurs at the point, $x=0.57 H$; that is, ahead of the wave crest. The crest position is given by $x=0$ or $n=0$.

In view of the substitutions from the eq 37, 39 , and 40 , and the transformation, $k x=n$, the formula for the rate of dissipation of energy, eq 23, reduces to

$$
\frac{d E_{1}}{d t}=-\frac{4 \mu A^{2}}{\sqrt{\pi k}} \sqrt{\frac{k \omega}{\nu}} \int_{-\infty}^{+\infty} Y(n) \operatorname{sech}^{2} n d n,
$$

which may be written also as

$$
\frac{d E_{1}}{d t}=\frac{4 A^{2}}{\sqrt{\pi}} \rho \sqrt{\frac{\omega \nu}{k}} N
$$

where

$$
N=\int_{-\infty}^{+\infty} Y(n) \operatorname{sech}^{2} n d n .
$$

The method of summations, selecting $n=0.1$, gave for $N$ the value 0.316 , or approximately $1 / \pi$. Using the value of $k$ from eq. 36 and $A$ from eq. 37 and remembering that $\omega=\sqrt{g H}$, the above expression for the rate of dissipation of energy per unit width of channel becomes

$$
\frac{d E_{1}}{d t}=4 \pi^{-3 / 2}\left(\frac{4}{3}\right)^{1 / 4} \rho \nu^{1 / 2} g^{5 / 4} h_{1}^{7 / 4} .
$$

As regards the energy of the solitary wave for unit width, we have

$$
E_{2}=\frac{8 \rho}{3 \sqrt{3}} g\left(H h_{1}\right)^{3 / 2}
$$

Differentiating with respect to $t$, and remembering that

$$
\frac{d h_{1}}{d t}=\sqrt{g H} \frac{d h_{1}}{d s},
$$

we find for the rate of decrease of the energy of the wave

$$
\frac{d E_{2}}{d t}=\frac{4}{\sqrt{3}} \rho g^{3 / 2} H^{2} h_{1}^{1 / 2} \frac{d h_{1}}{d s}
$$

By the principle of conservation of energy, the right-hand members of the eq. 42 and 43 are equal in absolute value, but they are of opposite signs. Making the comparison and rearranging the terms, we obtiin

$$
\left(\frac{h_{1}}{H}\right)^{-5 / 4} d\left(\frac{h_{1}}{H}\right)=-\frac{1}{3} \sqrt{\frac{\nu}{g^{1 / 2} H^{3 / 2}}} d\left(\frac{s}{H}\right) .
$$

This equation is derived by assuming that the channel is very wide, and, therefore, the effect of the vertical side walls can be neglected. If, however, the channel is narrow, the second term in the left-hand member must be multiplied by $[1+(2 H / B)]$, where $B$ denotes the width of channel. That is, in a channel of finite width,

$$
\left(\frac{h_{1}}{H}\right)^{-5 / 4} d\left(\frac{h_{1}}{H}\right)+\frac{1}{3}\left(1+\frac{2 H}{B}\right) \sqrt{\frac{\nu}{g^{1 / 2} H^{3 / 2}}} d\left(\frac{s}{H}\right)=0 \text {. }
$$

The integration gives, denoting the initial value of the crest height by $h_{10}$,

$$
\left(\frac{h_{1}}{H}\right)^{-1 / 4}-\left(\frac{h_{10}}{H}\right)^{-1 / 4}=K \frac{s}{H}
$$

where the coefficient of damping has the value

$$
K=\frac{1}{12}\left(1+\frac{2 H}{B}\right) \sqrt{\frac{\nu}{g^{1 / 2} H^{3 / 2}}} .
$$

This gives the variation of the crest height of a solitary wave with the distance traversed.

Comparing eq 33 and 44 , it is seen that the extinction of a limited wave takes place in a manner very different than it does with an unlimited wave. Although in the former case the extinction is characterized by a logarithmic decrement independent of height, this is not true of the solitary wave. The reason for this is to be found in the fact that the effective wavelength of a solitary wave is a function of the crest height. With 
494 First column. In equation above eq 4l, change $\sqrt{\pi} \bar{k}$ to $\sqrt{\pi} \mathrm{k}$. "In paragraph following equation $41, n=0.1$ should read, $\mathrm{d}_{\mathrm{n}}=0.1$.

$4 \$ 5$ Second column, lines 4 and $6 . h^{1}$ sholld be $h_{1}$. 
the decrease of the crest height, the effective length increases [4]. We may now accept the general statement that a logarithmic decrement independent of the wave height exists only for a wave that maintains a constant wavelength during extinction. Thus the damped oscillations of a liquid in a $U$-tube, of a standing wave in a rectangular basin and the damping of progressive oscillatory wave are characterized by a logarithmic decrement independent of the magnitude of the oscillations.

\section{John Scott Russell's Experiments on Solitary Waves}

To test the validity of the theoretical results above given, John Scott Russell's experimental data on solitary waves will be considered [5]. Although his tests were made to determine the law of velocity of propagation of solitary waves, the representation of data is such that they can be used also to determine the law of damping of waves. The experiments were made more than a century ago in a wooden rectangular channel, $1 \mathrm{ft}$. wide and $20 \mathrm{ft}$. long. Altogether some 55 tests are shown for various water depths and for various methods of wave generation.

Regarding the tests, Scott Russell states: "The original experiments are themselves given at the end of this paper for the purpose of enabling anyone who may be disposed to make use of them or any future purpose, either of framing or testing a theory, to make use of them much in the same way as if he had himself made the experiments." The farsightedness and disinterestedness of the investigator is evidence of a scientific attitude of the highest type and are cited by the present writer, to whom Russell's data have proven invaluable, as a model that other investigators might well follow.

Unfortunately the temperature of the water of the tests is not stated. Because of this, the desired comparison between theory and experiment will be made by deducing the temperatures of water from the observed damping of the waves and comparing these values with the prevailing air temperatures on the dates of the experiments.

In consulting the original data, it was seen that the tests could be grouped together and average values obtained for individual water depths $H$. The method of arriving at average values may be explained by considering the measurement for $H=$ $17.9 \mathrm{~cm}$. It was thought that five individual tests with this depth ought to be sufficient. The original data furnish values of $h^{1}$, the height of the wave, and $s$, the distance travelled. From these, the ratios $h^{1} / H$ and $s / H$ were formed and plotted. Since in these tests the initial heights $h_{10}$ were not the same, the plotting gave separate curves. Keeping one of the curves fixed, the others were shifted by different amounts parallel to the axis of abscissas so as to give a more or less congruent picture. The positions of the observed points after the shiftings are sbown in figure 4 . The centers of gravity of the points within selected increments of the abscissas were obtained and are given in table 2. Similar determinations were made for other depths and these are given likewise in table 2 .

TABLE 2. Eatinction of solitary wares; average values from Russell's experiments

\begin{tabular}{|c|c|c|c|c|c|}
\hline \multicolumn{2}{|c|}{$H=2.69 \mathrm{~cm}$} & \multicolumn{2}{|c|}{$H=7.61 \mathrm{~cm}$} & \multicolumn{2}{|c|}{$H=10.02 \mathrm{~cm}$} \\
\hline$s / H$ & $h_{1} / H$ & $s / H$ & $h_{1} / H$ & $s / H$ & $h_{1} / H$ \\
\hline 367 & 0.420 & 66 & 0.380 & 117 & 0.354 \\
\hline 535 & 265 & 255 & .305 & 424 & .286 \\
\hline 741 & .137 & 397 & .265 & 693 & .212 \\
\hline 962 & .112 & 605 & .197 & 930 & .168 \\
\hline 1,060 & .087 & 790 & .152 & 1,156 & .128 \\
\hline 1,170 & .057 & 950 & .130 & 1,398 & .099 \\
\hline 1,300 & .050 & 1,178 & .097 & 1,597 & .076 \\
\hline 1,502 & .055 & 1,470 & .067 & 1,742 & .060 \\
\hline 1,740 & .035 & 1,780 & .053 & 1,984 & .047 \\
\hline 2,100 & .020 & 2,270 & .043 & 2,503 & .030 \\
\hline \multicolumn{2}{|c|}{$\begin{array}{c}\text { Waves } 27,28,29 \\
\quad 31,33,34,35\end{array}$} & \multicolumn{2}{|c|}{$\begin{array}{c}\text { Waves } 25,26,40 \text {, } \\
\qquad 41,42\end{array}$} & \multicolumn{2}{|c|}{$\begin{array}{l}\text { Waves } 1,2,4,7 \text {, } \\
8,12,13,15,19,21\end{array}$} \\
\hline \multicolumn{2}{|c|}{$H=12.92 \mathrm{~cm}$} & \multicolumn{2}{|c|}{$H=15.80 \mathrm{~cm}$} & \multicolumn{2}{|c|}{$H=17.90 \mathrm{~cm}$} \\
\hline$s / H$ & $h_{1} / H$ & $s / H$ & $h_{1} / H$ & $s / H$ & $h_{1} / H$ \\
\hline 114 & 0.342 & 84 & 0.235 & 96 & 0.131 \\
\hline 388 & .262 & 351 & .181 & 291 & .110 \\
\hline 623 & 211 & 525 & .148 & 493 & .097 \\
\hline 765 & .174 & 657 & .128 & 683 & .087 \\
\hline 907 & .148 & 870 & .095 & 889 & .071 \\
\hline 1,088 & .125 & 1,085 & .076 & 1,097 & .063 \\
\hline 1,325 & .098 & 1,278 & .064 & 1,288 & .048 \\
\hline 1,672 & .068 & 1,435 & .054 & 1,494 & .045 \\
\hline 1,970 & .053 & 1,605 & .049 & 1,706 & .041 \\
\hline 2,515 & .027 & 1,790 & .042 & 1,910 & .032 \\
\hline \multicolumn{2}{|c|}{$\begin{array}{c}\text { Waves } \\
47\end{array}$} & \multicolumn{2}{|c|}{ Waves $48,49,50$} & \multicolumn{2}{|c|}{$\begin{array}{c}\text { Waves } 51,52,53 \text {, } \\
54,55\end{array}$} \\
\hline
\end{tabular}

The data for $H=17.9 \mathrm{~cm}$ from table 2 are plotted in figure 5. The smooth curve drawn through the points gives the adjusted values of 


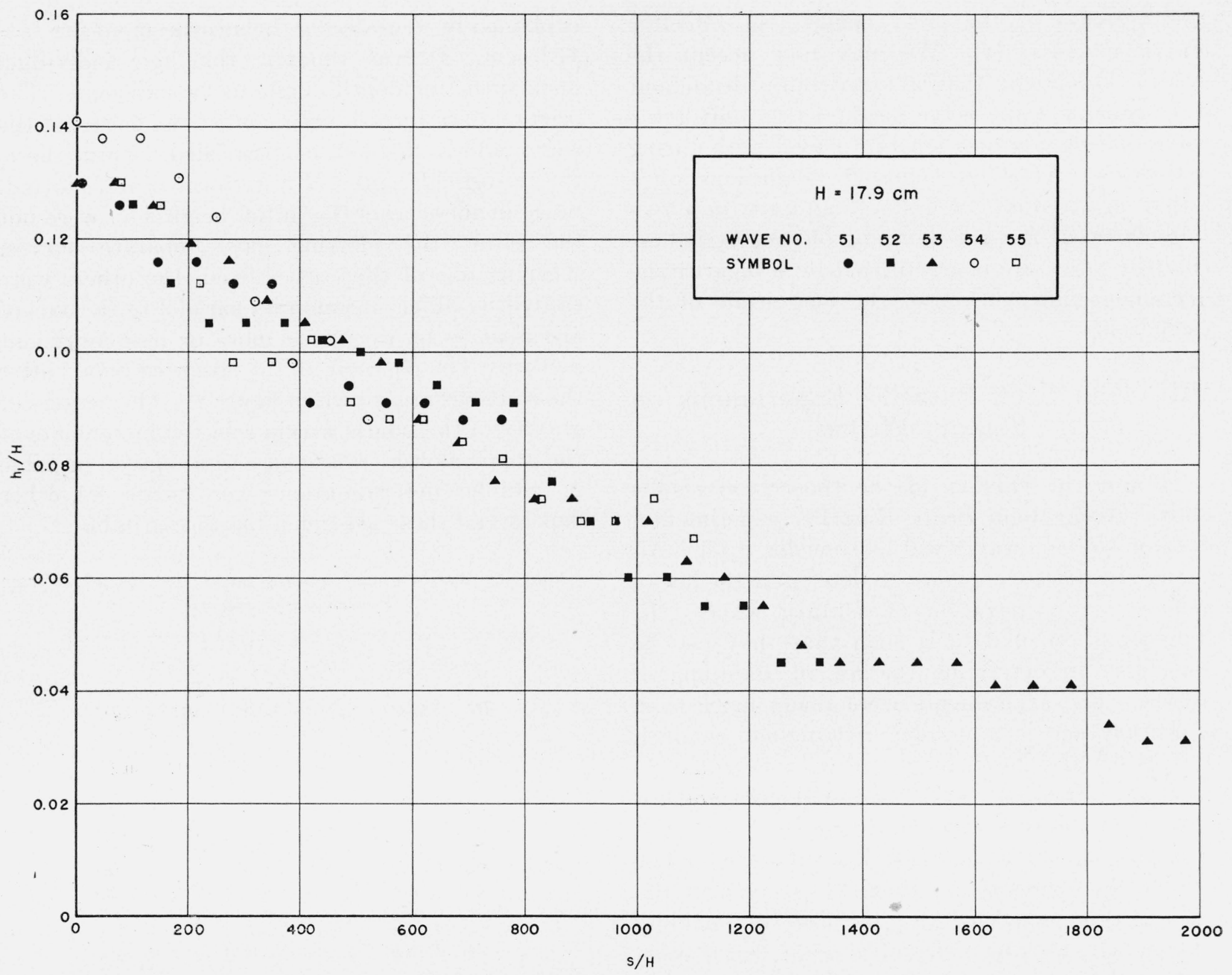

Figure 4. 'Data from Scott Russell's experiments on solitary waves.

the wave heights. The quantities $h_{1} / H$ and $s / H$ were read from the smooth curve. Values of the fourth root of $H / h_{1}$ were determined and are plotted in figure 6 with a convenient shift in the origin of $s / H$. The remaining data from table 2 were treated in a similar manner, and the resulting curves are shown in the figures 6 and 7 .

The distribution of the points is linear, a circumstance that accords very well with eq 44 . The slopes of the lines determine the coefficients of damping, $K$. The viscosities of the water can be obtained from eq 45, using the observed values of $K$, the corresponding values of $H$, and putting $B$, the width of flume, equal to $30.48 \mathrm{~cm}$. The individual determinations of water temperature are shown in table 3 , and the average value is found to be $15.8^{\circ} \mathrm{C}$.

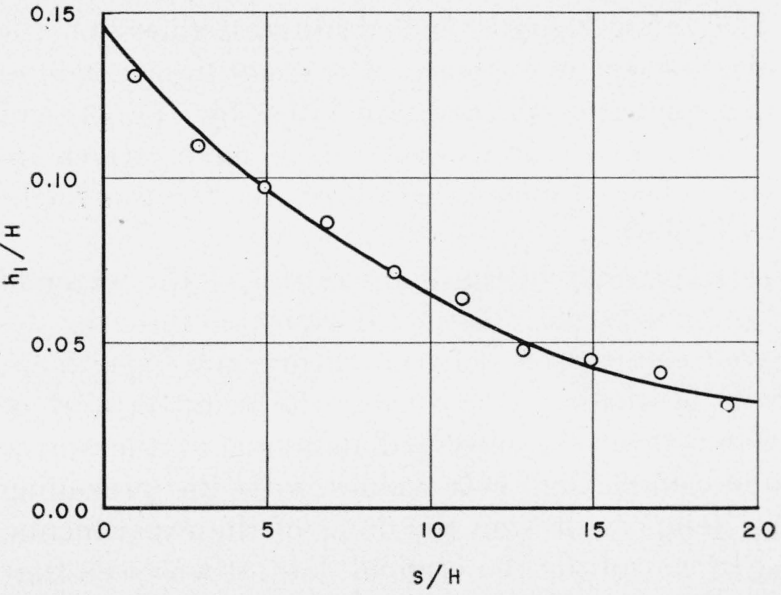

FIGURE 5. Decrease in height of a solitary wave with distanre traversed. $H=17.9 \mathrm{~cm}$ 


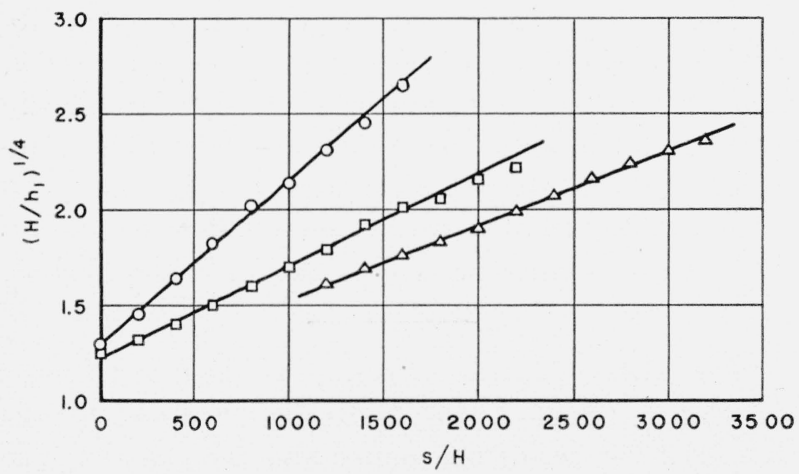

Figure 6. Coefficient of damping of solitary waves.

O, $H=2.69 \mathrm{~cm}, K=9.0 \times 10^{-4} ; \square, H=7.61 \mathrm{~cm}, K=4.85 \times 10^{4}$. $\triangle, H=17.90 \mathrm{~cm}, K=3.85 \times 10^{4}$.

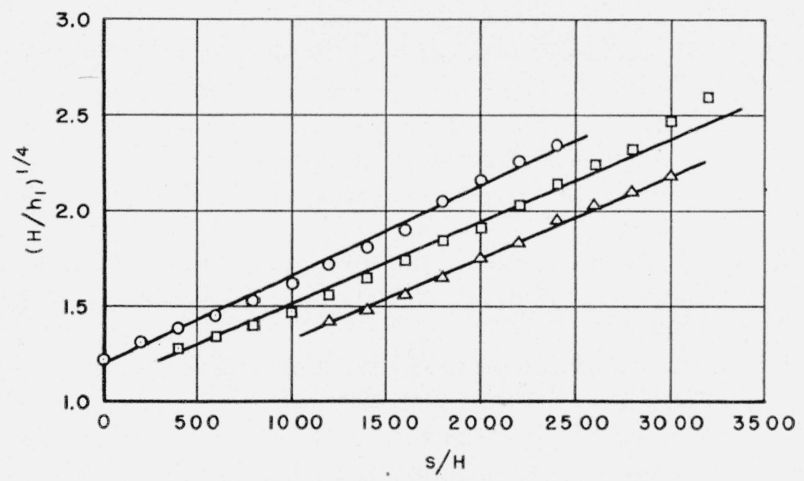

Figure 7. Coefficient of damping of solitary waves.

○, $H=10.02 \mathrm{~cm}, K=4.67 \times 10^{-4} ; \square, H=12.92 \mathrm{~cm}, K=4.25 \times 10^{4}$; $\triangle, H=15.80 \mathrm{~cm}, K=4.25 \times 10^{4}$

TABle 3. Determination of water temperatures in Russell's experiments

\begin{tabular}{|c|c|c|c|}
\hline$H$ & $K$ & $\nu$ & $\theta$ \\
\hline $\mathrm{Cm}$ & & & ${ }^{\circ} \mathrm{C}$ \\
\hline 2. 69 & $9.00 \times 10^{-4}$ & 0.01168 & 14. 2 \\
\hline 7.61 & 4. 85 & .01046 & 18.6 \\
\hline 10.02 & 4. 67 & .01133 & 15.4 \\
\hline 12. 92 & 4. 25 & .01132 & 15.4 \\
\hline 15.80 & 4. 25 & .01215 & 12.7 \\
\hline 17.90 & 3. 85 & .01048 & 18.5 \\
\hline
\end{tabular}

This average temperature of $15.8^{\circ} \mathrm{C}$ can be compared with the prevailing mean air temperatures at the time of the experiments. The Russell experiments were made in Edinburgh during the period, August 3 to 8, 1837. It may be said that when water has been introduced from an outside reservoir into a wooden flume of small depth inside a laboratory, during the latter months of summer, the working temperature of the water of the flume is best approximated by the morning air temperature, rather than the minimum or maximum daily air temperatures. Through the courtesy of the Meteorological Office, Air Ministry, Edinburgh, the air temperatures for the dates corresponding to Russell's experiments were communicated to the Liaison Officer, Meteorological Office, U. S. Weather Bureau, Washington, D. C. The data later were transmitted to the author by the Chief of the Division of Climatological and Hydrologic Services, U. S. Weather Bureau. The author wishes to express his appreciation of these favors. The Royal Society of Edinburgh have permitted a copy to be made of the pertinent part of Mr. Adie's original register for 1837, and the part of the data relating to the temperatures is given in table 4. It is the opinion of the Edinburgh Meteorological Office that the morning and the evening readings of the dry bulb were taken at $10 \mathrm{a} . \mathrm{m}$. and $10 \mathrm{p.m}$. Accordingly, the temperatures described as the morning temperatures should be close to the water temperatures of the Russell experiments. The average of the morning temperature for the 7 days is about $14.6^{\circ} \mathrm{C}$. This agrees reasonably well with the average temperatures obtained from the analysis of the wave data; that is, $15.8^{\circ} \mathrm{C}$.

TABLE 4.-Edinburgh temperatures, August 1837

\begin{tabular}{|c|c|c|c|c|}
\hline $\begin{array}{l}\text { Day of } \\
\text { month }\end{array}$ & Morning & Evening & $\begin{array}{l}\text { Mini- } \\
\text { mum }\end{array}$ & $\begin{array}{l}\text { Maxi- } \\
\text { mum }\end{array}$ \\
\hline & ${ }^{\circ} \mathrm{F}$ & ${ }^{\circ} \mathrm{F}$ & ${ }^{\circ} \mathrm{F}$ & ${ }^{\circ} \mathrm{F}$ \\
\hline 1 & 60 & 53 & 49 & 57 \\
\hline 2 & 55 & 53 & 50 & 55 \\
\hline 3 & 61 & 55 & 48 & -59 \\
\hline 4 & 52 & 49 & 48 & 57 \\
\hline 5 & 54 & 53 & 44 & 58 \\
\hline 6 & 56 & 54 & 46 & 60 \\
\hline 7 & 60 & 56 & 44 & 66 \\
\hline
\end{tabular}

The favorable agreement between the two temperatures and the fact that the inverse of the fourth root of the wave height varies linearly with the distance of the travel of the waves are proofs enough to show that the present theory of viscous action is sufficient to evaluate the wave dissipation for the water depths considered. The next step in the investigations of this type should be to determine what depths of water will result in a type of dissipation other than that caused by viscosity. 


\section{Application to Model Harbor Tests}

To the extent that the theoretical formula for the damping of solitary waves is satisfactory, at least when the waves are moving in shallow water, we can apply the results of the above theory to model tests of wave action. The models are of the type in which the depths of water involved are small and in which the pattern of the individual waves can be approximated as a succession of solitary waves. No serious error is made by replacing the ordinary pattern of the shallow-water oscillatory waves by a succession of solitary waves if this is done for the purpose of evaluating the energy dissipation. The method has the advantage that the wavelengths do not enter into the computations, so that the comparisons may be made on the basis of wave height only.

One problem that most readily suggests itself and is susceptible to approximate analysis is the model test of wave action in a harbor. Ordinarily the heights of the waves approaching a model harbor are determined on the basis of Froudian scales after the respective heights of the prototype waves have been selected. For the actual model these values of the initial wave heights must be increased over those determined by the Froude law in order to correct for the energy dissipation in the model harbor. In the prototype the dissipation is not large, so that it can be ignored, and this fact facilitates the determination of the proper model wave heights. The exact determination of the desired model wave heights in the approaches to the model harbor depends on the length and depth proportions of the harbor. The evaluation of the energy dissipation may be made step by step for various consecutive segments of the model. The average of the time rate of dissipation for the entire harbor area can then be evaluated. The height of the approaching waves is increased to such an extent that the increased flow of energy just balances the energy dissipation in the harbor.

The author expresses his appreciation to Herbert N. Eaton, Chief of the Hydraulics Section, for his editorial review of the paper and for constructive suggestions; also to George W. Patterson, his former colleague, for advice and the review of the mathematical methods used in the paper.

\section{References}

[1] J. Boussinesq, Complément à une étude intitulée: «Essai sur la théorie des eaux courantes》, et à un mémoire «Sur l'influence des frottements sur les mouvements reguliers des fluides $\gg$. Journal de mathématiques pures et appliqúees, série $3^{\mathrm{e}}, \mathbf{4}, 335$ (1878).

[2] A. G. Webster, Partial differential equations of mathematical physics, p. 172 (B. G. Teubner, Leipzig, 1927).

[3] G. H. Keulegan and G. W. Patterson, J. Research NBS 24, 47 (1940) RP1272.

[4] H. Lamb, Hydrodynamics, (ed 5) p. 401 (Cambridge University Press, Cambridge, Eng., 1924).

[5] Report of the Committee on Waves, appointed by the British Association at Bristol in 1836, and consisting of Sir John Robinson, Secretary of the Royal Society of Edinburgh, and John Scott Russell, Report of the seventh meeting of the Brit. Assn. Adv. Sci. p. 417 (John Murray, London, 1838).

Washington, January 27, 1948. 